Genetics, Maternal And Child Health (dinogmi), Departimento di Neuroscienze, Università di Genova, Genoa, Italy

${ }^{*}$ Corresponding author.

doi: 10.1192/j.eurpsy.2021.262

Introduction: Experience of bullying may be a significant risk factor for non-suicidal self-injury (NSSI).

Objectives: This study had three aims: to systematically investigate the association between bullying and NSSI, to analyze the possible mechanisms underlying the two phenomena, and to evaluate any differences between bullying victimization and bullying perpetration with respect to NSSI.

Methods: A systematic search about the association between bullying victimization and perpetration and NSSI was conducted using specific databases (PubMed, Scopus, Science Direct). The following keywords were used in all database searches: "bullying" AND "NSSI" OR "peer victimization" and NSSI.

Results: The searches in PubMed, Scopus and Science Direct revealed a total of 88 articles about bullying or peer victimization and NSSI. However, only 29 met our inclusion criteria and were used for the present review. Overall, all studies examined victimization; 4 studies also evaluated the effects of perpetration and 1 included bully-victims. According to the main findings, both being a victim of bullying and perpetrating bullying may increase the risk of adverse psychological outcomes in terms of NSSI and suicidality in the short and the long run.

Conclusions: To the best of our knowledge, this is the first review to systematically evaluate the relation between bullying victimization/ perpetration and NSSI. The main results support a positive association. Future research should evaluate the possible role of specific mediators/moderators of the association between experience of bullying and NSSI.

Disclosure: No significant relationships.

Keywords: Bullying perpetration; Bullying victimization; nonsuicidal self-injury; adolescents

\section{Comorbidity/dual pathologies}

\section{4}

Association between adult adhd and generalised joint hypermobility, with and without systemic manifestations: A case-control study

M. Glans ${ }^{1 \star}$, S. Bejerot ${ }^{1}$, M. Humble ${ }^{1}$, M. Elwin ${ }^{1}$ and N. Thelin ${ }^{2}$

${ }^{1}$ Faculty Of Medicine And Health, Örebro University, Örebro, Sweden and ${ }^{2}$ Division Of Psychiatry, Linköping University Hospital,

Linköping, Sweden

${ }^{\star}$ Corresponding author.

doi: 10.1192/j.eurpsy.2021.263

Introduction: There is growing evidence that generalised joint hypermobility $(\mathrm{GJH})$ is associated with several psychiatric conditions. There are no previous studies on adult ADHD.

Objectives: To evaluate, in a large Swedish sample, if generalised joint hypermobility and adult ADHD are associated.
Methods: 431 adults with ADHD and 417 controls were included. GJH was assessed by the Beighton Score, a physical examination, and the 5PQ, a self-report screening tool. Exploratively, reported musculoskeletal symptoms and abnormal skin manifestations suggestive of symptomatic GJH (e.g. Ehlers-Danlos syndrome), were assessed to differentiate this group from the general GJH group. Logistic regressions determined the influence of an ADHD diagnosis and known covariates (age, sex and ethnicity) on GJH and symptomatic GJH respectively.

Results: ADHD was associated to GJH, as defined by the Beighton Score and the 5PQ, with adjusted odds ratios of 4.65 (CI 95\% 3.017.18, $\mathrm{p}<.005$ ) and 1.86 (CI 95\% 1.39-2.48, p<.005), respectively. Likewise, ADHD and symptomatic GJH were associated with adjusted odds ratios of 6.94 (CI 95\% 4.05-11.89, $\mathrm{p}<.005$ ) and 2.66 (CI 95\% 1.94-3.66, $\mathrm{p}<.005$ ).

Conclusions: GJH and adult ADHD are associated conditions. Symptomatic GJH, defined as additional symptoms of pain and/or skin manifestations, has a considerably stronger link to adult ADHD than unspecific GJH has. GJH may represent a marker of an underlying systemic disorder with physical manifestations in connective tissue as well as behavioural manifestations including hyperactivity, impulsiveness and inattentiveness. Future studies should investigate if this represents a novel subtype of ADHD and if symptomatic GJH affects the ADHD management.

Disclosure: No significant relationships.

Keywords: comorbidity; Hypermobility; ADHD; biomarkers

\section{5}

Substance-specific variability of ADHD symptoms in riga psychiatry and addiction medicine centre treatmentseeking substance use disorder outpatient population

M. Ennīitis ${ }^{1 \star}$, M. Laizāne ${ }^{1}$, N. Bezborodovs ${ }^{2}$, I. Landsmane $^{3}$ and S. Skaida ${ }^{4}$

${ }^{1}$ Faculty Of Residency, Riga Stradins University, Riga, Latvia;

${ }^{2}$ Department Of Psychiatry And Narcology, Riga Stradins University, riga, Latvia; ${ }^{3}$ Department Of Addiction Medicine, Riga Psychiatry and Narcology Centre, Riga, Latvia and ${ }^{4}$ Department Of Psychiatry And Narcology, VSIA Riga Center of Psychiatry and Narcology, Riga, Latvia ${ }^{\star}$ Corresponding author.

doi: 10.1192/j.eurpsy.2021.264

Introduction: Studies show a link between attention deficit disorder (ADHD) and substance use disorders (SUD). Patients may abuse illicit drugs or alcohol as means of self-medicating e.g. stimulants for ADHD symptoms. Identifying ADHD symptoms in SUD patients could help improve treatment outcome and quality of life.

Objectives: To evaluate the prevalence of ADHD symptoms in Riga Psychiatry and Addiction Medicine Centre (RPNC) outpatients and study the link between ADHD symptoms and specific SUDs. Methods: In a period of 30 days, all consentient clients of RPNC outpatient addiction clinic were surveyed for basic sociodemographic data and screened with Adult ADHD Self-report Scale (ASRSv1.1). Results were compared among patients with different types of addictions previously diagnosed using ICD-10 classification. Results were also compared to a control group.

Results: Out of 279 participants, 209 were treatment-seeking SUD patients, 70 controls. Mean age was $37.99,77.8 \%$ were male. Among 